\title{
Rol de la universidad en la cooperación universidad-empresa- gobierno como un sistema regional de innovación: el caso Arequipa, Perú
}

\author{
Edgar A. Borda-Rivera ${ }^{1 *}$ y Gianny C. Ortega-Paredes ${ }^{2}$ \\ Universidad Católica de Santa María, Facultad de Ciencias Histórico Sociales, San Jerónimo S/N, Arequipa-Perú. \\ (Correo-e: ebordari@ucsm.edu.pe,gortega@ucsm.edu.pe)
}

Recibido Dic. 7, 2020; Aceptado Mar. 10, 2021; Versión final Jun. 2, 2021, Publicado Dic. 2021

\begin{abstract}
Resumen
El principal objetivo de esta investigación es evaluar el rol de la universidad en la cooperación (triple hélice) entre universidad, empresa, y gobierno en el sistema regional de innovación de Arequipa (Perú). Se entrevista a siete directores de escuelas de posgrado y se aplica una encuesta a 265 estudiantes de posgrado matriculados en el periodo 2017-2018 en el área de ciencias sociales (derecho, administración, proyectos de inversión, economía, y ciencias histórico sociales). Los resultados muestran un incremento significativo de producción de conocimiento y el inicio de redes de investigación con la empresa y el gobierno. Se observa un cambio del modelo económico que impulsa la investigación y la innovación según la demanda de productos de exportación tradicional y no tradicional, sin embargo, son las barreras culturales las que ralentizan la dinámica de la triple hélice. Se concluye que las universidades regionales han incrementado la cantidad y calidad de investigadores, así como la producción de publicaciones e inversión en investigación.
\end{abstract}

Palabras clave: sistema; innovación; triple hélice; investigación; universidad emprendedora

\section{The role of the University in the university-business- government collaboration as a regional innovation system: the case of Arequipa, Peru}

\begin{abstract}
The main objective of this research study is to assess the role of universities in the university-businessgovernment (triple-helix) collaboration in Arequipa's (Peru) regional innovation system. Seven graduate school directors are interviewed and a survey is applied to 256 social science (law, management, investment projects, economy, and history social science) graduate school students enrolled in the 2017-2018 year. The results show a significant increase in the production of knowledge and in the formation of new research networks between universities, businesses, and the government. There is also a change in the economic model in that research and innovation are enhanced according to demand for traditional and non-traditional export products. However, there are cultural barriers that slow down the dynamics of the triple-helix. It is concluded that regional universities have increased their quantity and quality of researchers, their research publications, and their investment into research.
\end{abstract}

Keywords: innovation system; triple helix; research; entrepreneurial university 


\section{INTRODUCCIÓN}

La primera revolución académica que protagoniza la Universidad se da a finales del siglo XIX en Europa, dando nacimiento a la segunda misión de la universidad, la investigación, sobre la base de las propuestas de Justus Liebig, inventor del "laboratorio de enseñanza" y de Willhelm von Humboldt, que integra la enseñanza y la investigación como paradigma académico; el papel del estado, es financiar las universidades de tal manera que se mantuviera su autonomía (Frølich, Coate, Mignot-Gerard y Knill, 2010), Universidad de doble misión, que servirá de inspiración y realización para la universidad norteamericana (Etzkowitz et al., 2020). La universidad será formadora de académicos y también se convertirá en el centro de especialistas en investigación. Los estadounidenses unieron la idea universitaria británica con el énfasis de la investigación alemana y el concepto de escuela de posgrado con las nociones populistas estadounidenses y así crearon la universidad estadounidense contemporánea (Altbach, 1989).

En la segunda mitad del siglo XX, la universidad protagoniza su segunda revolución académica, dando lugar a la tercera misión de la universidad, el emprendedurismo, con relevancia del conocimiento y la investigación para el desarrollo económico en el mundo. Esta nueva función plantea retos institucionales intra y extra universitarios, como los cambios de la institución universitaria, resultado de las iniciativas de docentes y estudiantes involucrados en la investigación, dando lugar a una nueva estructura académica en las universidades; destacando entre otras, la universidad de Stanford que protagoniza importantes cambios curriculares y organizativos, con base en la actividad investigativa de estudiantes y profesores. Según Etzkowitz, entre 1890-1900, los graduados forman empresas para desarrollar la infraestructura tecnológica de la región; en 1920 se organizan pasantías de estudiantes en empresas locales; en 1930 gestionan patentes; en 1940 inicia nuevas empresas basadas en la innovación tecnológica; en 1950 se convierte en proveedor de I + D de electrónica para el gobierno federal y para mejorar las capacidades académicas internas e industrias regionales; en 1970 apertura una unidad administrativa para gestionar la transferencia de tecnología y curso interdisciplinario para resolver problemas empresariales; en 1980-1990 incluye en el plan de estudios de pos y pregrado el emprendimiento; en el 2010 se fomenta la formación de inicio en innovación. El surgimiento del laboratorio de investigación industrial y la cientificación de la producción industrial habían creado un mercado laboral para académicos capacitados experimentalmente. Las universidades ofrecen un lugar específico para la integración y diferenciación entre funciones en la infraestructura del conocimiento como el aprendizaje académico, la teorización y las prácticas experimentales, creándose un nuevo contrato social entre la universidad y la sociedad en general. El contrato anterior se basó en un modelo lineal de innovación, suponiendo solo contribuciones a largo plazo del conocimiento académico a la economía. Ahora se ven posibles contribuciones a corto y largo plazo, basadas en ejemplos de formación de empresas y contratos de investigación en campos como la biotecnología y la informática (Etzkowitz y Leydesdorff, 1995).

Esto implica diferentes tipos de conocimiento, de los cuales los menos formalizados, aprendidos a través de la experiencia, son a menudo tan importantes como los formalizados, aprendidos a través de la exposición a la enseñanza. La universidad ya no puede seguir organizada según las disciplinas y métodos educativos tradicionales, los principios y culturas educativos de colaboración, interdisciplinariedad y de compromiso con los problemas de la vida real son claves (Lundvall et al., 2008). cobrando más importancia el "aprendizaje relacional" y la formación en "habilidades personales". se trata de un aprendizaje que se realiza a través del hacer, del usar y de la interacción (denominada aprendizaje DUI), para ello se debe crear un mercado de conocimiento implícito, lo que significa establecer una interacción más estrecha con la sociedad, y dice Lundvall (2016), esto es especialmente importante en los países menos desarrollados, donde la distancia entre la academia y la vida real suele ser muy grande, además, recomienda tomar en cuenta las siguientes tres tendencias en el pensamiento del desarrollo: Primero, enfoque creciente en las capacidades más que en la dotación de recursos como los principales instrumentos y valores en el desarrollo (Sen, 1999). Segundo, el conocimiento como el recurso quizás más crucial que impulsa el desarrollo. Tercero, las instituciones como las "causas fundamentales" del desarrollo, empequeñeciendo la importancia de todos los demás factores, como la geografía y las políticas (Lundvall, 2007).

Estos nuevos cambios son más complejos y difíciles en los países latinoamericanos debido a su historia colonial que a la fecha están presentes en la cultura de la enseñanza y el aprendizaje, por lo que es inevitable resolver estos desafíos que enfrenta a las personas y las organizaciones con nuevas demandas (Lundvall, 2008). La universidad debe promover y formar en los estudiantes nuevas habilidades generales en comunicación, cooperación y creatividad, para estimular la creatividad colectiva se usa como estrategia el trabajo en equipo y resolver problemas no estructurados con un maestro como entrenador (Lundvall, 2016). De esta forma los cursos de las especialidades sociales y humanidades como historia general (social, política y cultural), historia económica y La historia industrial se hacen indispensables y los más importantes, como ya lo afirmaba Schumpeter (1939), todos los demás materiales y métodos, estadísticos y teóricos, sólo están subordinados a ellos y sin valor sin ellos (Lundvall, 2008). 
Cook (1997), añade que en este proceso juega un rol estratégico fundamental la cultura, porque la innovación y el aprendizaje están estrechamente vinculados, no puede haber cambio sin un aprendizaje previo; reconociendo que se aprende haciendo, usando y por interacción, añade que el aprendizaje está vinculado a una determinada estructura institucional y requiere de un cambio institucional, cultural, de reglas, hábitos, etc, y finalmente señala que el aprendizaje requiere medios, incentivos y la capacidad de los individuos o sistemas para adquirir este conocimiento. Este conjunto de cambios representa un cambio cultural "La cultura social y tradicional de un lugar se transforma en una cultura técnica a través de la orientación tecnológica del sistema de educación y formación, una cultura que es humanista pero que se enriquece con habilidades tanto explícitas como tácitas", porque la política de innovación implica la creación de un clima y determinadas actitudes que permitan la coordinación entre los agentes encaminados a lograr la innovación. A esto lo denomina "cultura de la cooperación", que exige una "voluntad de aprender y compartir información" (Cooke et al.,1997). Universidades e industria están asumiendo tareas que antes eran en gran parte competencia del otro, igualmente el rol del gobierno está cambiando, están ofreciendo incentivos, por un lado, y presionando a las instituciones académicas, por el otro, para que vayan más allá de realizar las funciones tradicionales de memoria cultural, educación e investigación, y hagan una contribución más directa a la "creación de riqueza" (Etzkowitz y Leydesdorff, 1995); las universidades empiezan a crear una nueva infraestructura de conocimiento emergente.

Las nuevas formas de interacción de estos tres actores, está dando inicio a la triple hélice que responda aún modelo en espiral de innovación para capturar múltiples vínculos recíprocos en diferentes etapas de la capitalización del conocimiento (Etzkowitz y Leydesdorff, 1995); las instituciones de estos actores enfrentan el reto de construir nuevas políticas de CTI que faciliten el desarrollo industrial junto al desarrollo científico tecnológico, que han dado lugar a "modelos coevolutivos", que analizan los desarrollos en términos de redes; a sistemas de innovación nacional, que se complementan con sistemas de innovación regionales, locales y multinacionales, como es el caso de la Unión Europea; el estado promueve políticas tecnológicas, prácticamente en todas las regiones, independientemente de que sean intensivas en investigación o industrialmente, también los programas internacionales y multinacionales de la ONU, la OCDE, el Banco Mundial, el BID y la Unión Europea ayudan al desarrollo económico confiando en las relaciones entre la academia, la industria y el gobierno para lograr sus objetivos (Etzkowitz y Leydesdorff, 1995). Los aportes de Porter, (1995) así como la noción de "triple hélice", en la década de 1990 (Leydesdorff y Etzkowitz, 1996, 1998; Etzkowitz y Leydesdorff, 1997, 1998), sirven para construir el modelo de la 'triple hélice' como una forma de proporcionar una mayor comprensión de la dinámica compleja entre tres tipos de actores: gobierno, empresas y centros de conocimiento (Looy et al., 2003). Cuanto mayor sea el carácter 'emprendedor' demostrado por estas instituciones, mayor será el impacto positivo en el desarrollo de una región en términos de su desempeño en innovación (Porter, 1995).

Desde su aparición, el concepto de "triple hélice" ha evolucionado con distintos significados, primeramente en la Triple Hélice I, el estado nacional abarca la academia y la industria y dirige las relaciones entre ellas, con muy poco espació para iniciativas "de abajo hacia arriba", la innovación se desalentó en lugar de alentarla; la Triple Hélice II conlleva una política de laissez-faire y la Triple Hélice III, tiene como objetivo común realizar un entorno innovador compuesto por empresas derivadas de universidades, iniciativas trilaterales para el desarrollo económico basado en el conocimiento y alianzas estratégicas entre empresas grandes y pequeñas, que operan en diferentes áreas y con diferentes niveles de tecnología, laboratorios gubernamentales, y grupos de investigación académica (Etzkowitz y Leydesdorff, 2000). Se propone que las universidades nacionales pueden estar vinculadas a través de acuerdos de redes de colaboración con las universidades regionales y el reglamento de los profesores incluyen la posibilidad de trabajar en ambas para brindar a las universidades regionales conocimientos actualizados sobre la investigación de frontera. Se debe estimular a las universidades regionales para que desarrollen investigación avanzada en nichos donde revelen fortaleza o en áreas donde la demanda regional sea fuerte (Lundvall, 2008).

La tercera misión de la universidad no cambia su esencia formadora y educativa, sino que se reinventa para atender las nuevas necesidades del siglo XXI, tampoco pierde sus ventajas comparativas frente a las corporaciones y las consultoras que se dedican a la investigación. La universidad sigue siendo la institución central del sector del conocimiento siempre que mantenga su misión educativa original Etzkowitz, Webster, Gebhardt y Terra. La docencia es la ventaja comparativa de la universidad, especialmente cuando está vinculada a la investigación y el desarrollo económico. Los estudiantes también son inventores potenciales, representan un flujo dinámico de "capital humano" en los grupos de investigación académica, en contraposición a los más estáticos. En comparación con otros aspirantes al liderazgo del conocimiento propuestos recientemente, como la empresa consultora, hay marcadas diferencias; la empresa consultora reúne personal ampliamente disperso para proyectos individuales y luego los dispersa nuevamente después de que se completa un proyecto, que resuelve el problema particular de un cliente. Estas empresas carecen de la capacidad organizativa para seguir un programa de investigación acumulativa como algo natural. Las ventajas comparativas únicas de la universidad es que combina la continuidad con el cambio, la memoria 
organizativa y de investigación con nuevas personas y nuevas ideas, a través del paso de generaciones de estudiantes (Etzkowitz y Leydesdorff, 2000).

Las nuevas políticas de CTI en los países desarrollados, a partir de la década de 1980 se concentran en formular políticas para construir un sistema de innovación nacional (SIN) como respuesta a los nuevos retos de la globalización, el neoliberalismo y el uso masivo de las TIC. EI SIN está compuesto por una serie de sectores, dentro de los que destaca el gobierno, la universidad, la industria y su entorno (DE CIENCIA, A. L. P., 2015). La innovación se constituye en el eje central de la industria en un mundo que se globaliza aceleradamente y los países desarrollados, para mantener y/o extender sus mercados, se preocupan en construir ecosistemas de innovación nacional, donde las industrias puedan construir una red de instituciones públicas y privadas, involucrando a la academia en permanentes procesos de innovación tecnológica. El concepto de sistema de innovación nacional fue introducido hacia finales de la década de 1980 por Freeman (1987), y otros autores como Lundvall (1992), Freeman (1987), conceptúa el SIN como una red de instituciones en los sectores público y privado cuyas actividades e interacciones inician, importan, modifican y difunden nuevas tecnologías. Todos los conceptos sobre SIN tienen como característica común referirse a la interacción en el marco de la producción, difusión y uso de conocimientos para la innovación. Sobre esta base, se plantean las siguientes interrogantes de investigación ¿Cuál es el rol de la universidad en la cooperación universidad, empresa, gobierno en el sistema regional de innovación Arequipa?, ¿Cómo ha afectado el cambio de modelo de sustitución de importaciones en las relaciones del sector académico y la industria en Perú-Arequipa? y ¿Cuáles son los avances y desafíos de la universidad en el desarrollo de su tercera misión cómo actor del sistema regional de innovación?

\section{METODOLOGIA}

La presente investigación se fundamenta en el método de estudio de caso a través de la investigación cualitativa, que permite una investigación holística y da sentido característico a los eventos de la vida real, con una visión pluralista (Yin, 1994); además, es algo específico, complejo en funcionamiento, un sistema integrado. Según Stake (1999), permite aprender sobre un problema general en un caso particular, por ello es un caso intrínseco, como es la región Arequipa. Esta metodología permite comprender particularidades temporales y espaciales de contextos locales (Rivera Polo y Rivera Vargas, 2018) de la universidad regional en Arequipa. El análisis de la universidad regional se enmarca en una visión de complejidad del desarrollo regional, porque "permite ver los hechos reales dentro de un contexto, dentro de una globalidad multidimensional y dentro de su propia complejidad (...) por lo que deben ser analizados tomando en cuenta los referentes culturales, los entornos socio políticos" (Guadián-Fernandez, 2010).

Las técnicas de recolección de información primaria fueron las entrevistas semiestructuradas en profundidad a informantes claves, siete directores de las escuelas de posgrado de la Universidad Nacional de San Agustín de Arequipa (UNSA), Universidad Católica de Santa María (UCSM) y Universidad Católica San Pablo (UCSP), en su gran mayoría fueron preguntas de opinión y en menor medida preguntas para recabar información sobre algún aspecto específico sobre investigación, su duración promedio fue de 40 a 50 minutos y se grabaron bajo el consentimiento de las personas entrevistadas, quienes manifestaron su acuerdo en hacer público su papel de informantes. Se aplicó una encuesta a 265 estudiantes de posgrado matriculados en el periodo 2017-2018 del área de ciencias sociales (Derecho, Administración, Proyectos de inversión, Economía, Ciencias Histórico Sociales) de la universidad regional. El proceso de análisis de los datos recogidos, se realiza siguiendo las pautas de Strauss y Corbin (2003), con un momento descriptivo y un momento relacional de codificación de los datos, para organizarlos, sintetizarlos, conceptualizarlos y relacionarlos. El momento descriptivo se realizó mediante un proceso de codificación abierta, identificando el concepto de investigación, así como sus propiedades relacionadas al tipo, propósito de la investigación y en una dimensión temporal 2017-2018. Mediante la codificación axial se relacionó la categoría investigación, innovación y emprendimiento, políticas de CTI, con subcategorías relacionadas a las líneas de investigación, las propuestas de la agenda de Arequipa, producción de paper, categoría de investigadores, proyectos de innovación y emprendimiento, empresa y gobierno como componente de la triple hélice. Este proceso permitió pasar de la descripción de los datos a definir el rol de la universidad en la innovación tecnológica de la región en la triple hélice regional.

La técnica de recolección de información secundaria fue la observación estructurada de la documentación oficial de las políticas; documentación de normativa jurídica, administrativa y documentación de fuentes estadísticas nacionales y regionales, análisis de 1,185 tesis de las escuelas de posgrado de la región Arequipa registradas en el repositorio de cybertesis, catálogo de biblioteca y repositorio Renati, correspondientes al periodo 2011-2017. La documentación sobre política, plan, agenda, evaluaciones, diagnósticos, memorias, sistematizaciones, informes, reportes. La documentación de fuentes estadísticas del Instituto Nacional de Estadística e Informática (INEI), de encuestas sobre temas específicos (Canon minero). Así mismo, se consultaron libros y artículos de revistas que aportan valiosos elementos conceptuales y metodológicos en el proceso de investigación, según los objetivos propuestos. Se exploró documentos relacionados al sistema de 
innovación regional a través de las agendas, líneas de investigación, proyectos y documentos oficiales de apoyo y respaldo de las autoridades académicas y políticas. Sobre la actividad investigativa y de enseñanza de la Universidad regional, paper publicados, documentos académicos sobre el capital humano avanzado y las normas que rigen a los Vicerrectorados de investigación.

Los datos recogidos en la encuesta fueron procesados con el instrumento SPSS y se presentaron en tablas de distribución de frecuencia, permitiendo un análisis estadístico básico. La información secundaria contenida en los diversos documentos fue sometida a un análisis de contenido, especificando unidades de registro coherentes a las categorías y sub categorías establecidas, distinguiendo unidades de muestreo como son las leyes que oficializan las políticas de CTI, las normas que rigen la vida universitaria, las investigaciones sobre el tema de estudio y otros; en segundo lugar se consideraron unidades de registro, relacionado a los diferentes temas de investigación y finalmente las unidades de contexto que han permitido caracterizar las unidades de muestreo y de registro. El tipo de validez usado en esta investigación corresponde a la triangulación de las fuentes de datos y metodológica (Guadián-Fernandez, 2010).

\section{RESULTADOS}

El Perú durante la primera década del nuevo milenio tuvo un crecimiento económico extraordinario de un promedio de $6 \%$ anual, desde el 2005-2016, lo que permitió un progreso social sin precedentes, bajando la pobreza de un $42 \%$ (2007) al 20\% (2018) (INEl, 2018), sin embargo, este crecimiento no es resultado del incremento de la productividad, de un proceso permanente de innovación. El modelo de sustitución de importaciones, de una economía cerrada consolidó una etapa de parálisis en investigación, en la producción de ciencia y tecnología de las universidades, las que por historia y cultura no están organizadas para producir conocimiento. La universidad latinoamericana nace bajo el modelo de la universidad española (En 1551 se funda la Universidad de San Marcos de Lima), fuertemente arraigada en el dogma y la formación eclesiástica, "con una enseñanza arcaica, verbalista y con un círculo cerrado que cuidaba las cátedras y sus dependencias como un bien de familia". En el año 1918 tiene lugar la "Reforma de Córdoba", con postulados estrictamente académicos y sociales, sin considerar la segunda misión investigativa de la universidad: autonomía universitaria, elección de los cuerpos directivos de las instituciones y otros (Del Huerto, 2018). El concepto de "libertad de catedra" de esta Reforma tiene un significado académico de libertad de enseñanza, más no de libertad de investigación al estilo de la universidad alemana.

La universidad desde su nacimiento no retoma la actividad científica nativa de las grandes culturas incas y preincas, como, por ejemplo, la biotecnología natural de animales (como la riquísima variedad de alpacas con diversos colores naturales) y vegetales (la gran variedad de alimentos como la papa, cereales, etc). El idioma inglés, es un factor condicionante, que tiene el monopolio de la distribución internacional del conocimiento científico. Al menos la mitad de las 100,000 revistas académicas del mundo se publican en inglés. La mayoría de las bases de datos están en inglés (Altbach, 2018). A pesar de que, en el año 1992, se establece un modelo de economía abierta en el Perú, de corte neoliberal, la élite gobernante peruana no tiene interés en la triple hélice, antes bien, deteriora la calidad universitaria, no promueve la investigación, mercantiliza la formación universitaria con el Decreto Legislativo $\mathrm{N}^{\circ}$ 882-1996, que apertura universidades privadas con fines de lucro, constituyendo una gran presión contra la producción del conocimiento, no intenta implementar el artículo 14 de la constitución de 1993 "la educación promueve el conocimiento, el desarrollo científico y tecnológico del país"; la violencia política de 20 años (1980-2000), hace que las universidades públicas sean consideradas escenarios de guerra contrasubversiva por el gobierno de turno, lo que las mantiene en intervención militar y administrativa a través de comisiones reorganizadoras, las que subsistieron hasta noviembre del 2000 (Vela Meléndez et al.,2018).

A partir del 2000, la investigación avanza muy lentamente, haciendo difícil establecer la triple hélice en el Perú, porque debe vencer retos como, la limitada disposición de recursos financieros, la existencia de monopolios y las barreras económicas al ingreso de nuevas empresas; las restricciones legales, las leyes, normas y reglamentos que buscan luchar contra la corrupción y la malversación de recursos públicos, hacen difícil que un organismo público haga negocios con organizaciones privadas; al interior de la propia universidad, los académicos pueden ver como indebido o ilegítimo el que otros profesores o la misma universidad, comercialicen el conocimiento; falta de especialistas en la transferencia tecnológica; falta de interés del sector privado por invertir en conocimiento, el sector empresarial prefiere importar tecnología (Ismodes, 2015). Los investigadores sostienen que el Perú necesita "un desplazamiento significativo de las políticas públicas, reorientándolas hacia la creación de capacidades en ciencia, tecnología e innovación (CTI) para avanzar hacia la sociedad del conocimiento", y de esta manera disminuir la dependencia de la exportación de productos primarios y avanzar hacia una economía intensiva en conocimiento e innovación capaz de atraer inversiones, creatividad y talento (Sagasti, 2014). El número de publicaciones de investigadores peruanos es muy modesto en comparación con los países de la región. En el año 1996 Perú produce 200 paper y en el 2005 menos de 150; en cambio Chile sube de más de 600 a 1,559 en el 2005, Argentina produjo 3,058. En Perú, por cada mil integrantes de la PEA hay solo 0.2 investigadores, nivel que 
se ubica por debajo del promedio de América Latina y el Caribe (1.3) y muy lejos del promedio de los países de la OCDE (12.7) (CONCYTEC, 2016). Las exportaciones, en los últimos diez años (2009-2018) las de alto, medio y bajo contenido tecnológico representan conjuntamente sólo el 9\%; en cambio, las de ningún grado de contenido tecnológico, manufacturas basadas en recursos naturales y otros representan el $91 \%$ (CIEN, 2020)

El SIN, en el Perú se crea el año 2005 con la denominación Sistema Nacional de Ciencia, Tecnología e Innovación Tecnológica (SINACYT), y el CONCYTEC como su organismo rector, que a la fecha solo está presente como una denominación. En los departamentos también se han dado iniciativas para construir sistemas de innovación regional, que no se ha logrado implementar. Su creación se hace en condiciones muy limitadas, sin el compromiso duradero del gobierno con la educación científica, la investigación y la infraestructura relacionada con la CTI, sin normas claras y amplias para la protección de la propiedad intelectual, tampoco se tiene confianza en las fuerzas del mercado como motor para la transferencia de tecnología. Las empresas no se preocupan por el desarrollo de la CTI. Una gran limitante es la falta generalizada de una cultura favorable a la investigación, el desarrollo y la innovación. La misma universidad tiene una limitada infraestructura tecnológica y un capital social avanzado en crecimiento. De trece instituciones estudiadas a nivel nacional en el año 2014, sólo tres de ellas, Universidad Peruana Cayetano Heredia (UPCH), Universidad Nacional Mayor de San Marcos( UNMSM) y Instituto Nacional de Investigación y Capacitación de Telecomunicaciones (INICTEL-UNI), tienen una unidad dedicada oficialmente al tema de Transferencia de Tecnología (TT), la Universidad Nacional Agraria La Molina (UNALM), está iniciando el proceso para reglamentar el funcionamiento de una unidad de TT y en lo regional sólo Arequipa, incorpora en su organización una unidad que realiza actividades de TT. El resto de instituciones, si bien realizan actividades de TT, estas se desarrollan de manera diversa y desde diferentes unidades que no necesariamente coordinan entre sí. Los organismos públicos de investigación (OPI), con muy pocos recursos y con una estructura legal muy rígida han generado conocimiento útil y valioso para pequeños productores a lo largo de todo el país.

Las universidades, destacan sus esfuerzos recientes por crear oficinas o unidades de TT de manera descoordinada como el Centro de Investigación de la Católica (CICA), en Arequipa, que labora en cooperar con los profesores, los alumnos, los laboratorios y unidades de investigación y para ello cuenta con un pequeño equipo de apoyo, realiza una labor de vinculación y de promoción del emprendimiento. Aunque no se cuenta con incubadora de empresas formal, se apoya a los alumnos interesados en la creación de empresas. La universidad, desde 1996-2014, logró 16 patentes, algo parecido sucede con las Spin Off, donde en la capital solo tres de ellas realizan actividades de incubación (PUCP, UPCH y UNMSM). sin casos de éxito destacados y no tienen un componente tecnológico. A pesar de que en el país se ha desarrollado un sistema económico de corte neoliberal desde el año 1992, hay poca vinculación entre la empresa y la universidad, y no existe un mercado alrededor del conocimiento (Ismodes, 2015). La primera experiencia de investigación de universidad - empresa, que permite un significativo desarrollo de capital humano avanzado en el Perú, se da con el programa de Fomento de la Innovación Tecnológica y la Competitividad en la Agricultura del Perú (INCAGRO), que inició en 2001 por iniciativa del Ministerio de Agricultura y cofinanciamiento del Banco Mundial y del Gobierno del Perú (Sagasti, 2014).

El año 2015, constituye un año de quiebre, la presión de los empresarios exportadores de productos no tradicionales al demandar financiamiento del Estado para proyectos de investigación, permiten establecer un conjunto de factores favorables a la triple hélice en el Perú y la región, como son, algunas normas estatales de inversión del Estado en CTI. El estado inicia un proceso de convocatorias para la innovación, emprendimiento, incubadoras, startup, para la empresa, pero a la universidad todavía no se le reconoce como institución que pueda tener funciones semejantes a una empresa. Entre los años 2015-2020 se llevan a cabo 17 convocatorias, de las cuales en tres de ellas la universidad tiene la posibilidad de presentar un proyecto como solicitante, en siete programas participa como asociado y en otros siete programas no figura (innóvate Perú, 2015-2020). La empresa, se incorpora lentamente y con dificultades en participar con cofinanciamiento en programas de innovación, el $4 \%$ de las empresas que realizaron innovación lo hicieron con financiamiento del estado, el $25 \%$ del total de empresas que conocen de los programas y servicios públicos de apoyo a la innovación postularon a ellos, el $61 \%$ de las empresas que conocían de los programas y servicios y que no postularon considera que estos no se adaptan a sus necesidades, el $39 \%$ de las empresas que conocían de los programas y servicios no postularon por dificultades burocráticas, el $11 \%$ de los beneficios tributarios ofrecidos para proyectos en $\mathrm{I}+\mathrm{D}+\mathrm{i}$ fueron aprovechados en 2017 (consejo nacional de competitividad y formalización, 2019). Se logra finalmente, en el año 2016, hacer uso del "canon minero", que es una gran fuente de financiamiento para la CTI.

El cambio del modelo cerrado de sustitución de importaciones, por un modelo abierto, y la reinstalación democrática a partir del año 2001, permitió un extraordinario crecimiento de las exportaciones tradicionales del país, de 4,829 millones de dólares del 2000 a 37,000 millones de dólares en el 2018 (18 veces más), y las no tradicionales, de 2,000 millones de dólares a 13,000 millones de dólares (6 veces más), impulsando la 
necesidad de la investigación y la innovación exigida por la demanda del mercado mundial, permitiendo iniciar la construcción de la triple hélice regional, cuyo avance es ralentizado por estar aún vigente la cultura de la desconfianza, la baja cooperación y la discriminación. La nueva Ley universitaria permite cambios importantes en la universidad regional, como el incremento del número de investigadores en un $415 \%$ (pasando de 454 a 1,676 investigadores) del 2016 al 2020 (Registro Nacional de Ciencia), se crea el Vice rectorado de investigación, entre otros, lo que permite aumentar la producción de paper publicados del 2014 al 2019, de 547 a 1,833 (Scimago, 2019). La inversión en CTI a partir del 2016 se presenta más favorable; el mismo CONCYTEC mejora su rol y desempeño aprendió a superar tres grandes obstáculos, primero, la indiferencia de las autoridades políticas, que muchas veces simplemente no entienden estas políticas, segundo, la gran ignorancia sobre las características y el contenido de dichas políticas por parte de los funcionarios encargados de diseñarlas y tercero, la incompetencia de gestores a cargo de poner éstas políticas en práctica (Sagasti, 2014).

La universidad privada sin fines de lucro, como la UCSM, también fortalecen sus actividades de CTI, de tener 300 mil nuevos soles anuales hasta el 2015, pasa a un presupuesto interno de 6 millones de nuevos soles en el 2019. La UCSP, invierte en proyectos de I+D+i junto a fondos concursables del CONCYTEC, de 1,361,100 nuevos soles en el 2010, a 9,349,635 nuevos soles en el 2016 (UCSM, UCSP). La universidad regional cuenta con tres incubadoras (Jaku, Kaman e Inicia), desde el año 2014, que ha permitido iniciar, nuevas pequeñas empresas, la mayoría de base tecnológica participando en distintas convocatorias del Estado. Los Centro de Innovación Productiva y Transferencia Tecnológica (CITE), creados por el Estado el año 2002, intervienen activamente en el sector agroindustrial en Majes, lleva acabo importantes proyectos de investigación e innovación, junto con las universidades de la región, para elevar la productividad de los pequeños empresarios agrícolas y facilitar la exportación de productos no tradicionales.

Solo dos grandes empresas de la región han iniciado esta participación, Incalpaca Textiles Peruanos de Export, la cual utilizó tecnología de visión artificial para obtener hilados de alta calidad; luego la empresa de fabricación de ladrillos de construcción a partir del uso de relaves mineros, a cargo de Cepromet Minera Porvenir S.A.C., e IT Software. La institución empresarial más antigua de la región, la Cámara de comercio e industria de Arequipa ha formalizado la creación de un parque científico tecnológico con el propósito de promover la innovación tecnológica en coordinación con el municipio de Arequipa y las universidades. Las industrias tradicionales de la región Arequipa son las procesadoras de fibra de alpaca, lo exclusivo de esta industria es que la fibra de alpaca solo existe en los andes centrales de Sudamérica, y el Perú es el eje de esta región y dentro de Perú, Arequipa es la que ha desarrollado la industrialización de esta fibra. El 96\% de las principales empresas exportadoras de pelo fino de alpaca se encuentra en la región Arequipa (MINAGRI, 2020). Sin embargo, tradicionalmente estas empresas no solo se desarrollan de manera divorciada de los centros tecnológicos y académicos de la región, sino que además, desde su fundación ha usado tecnología extranjera, incluso a pesar de que en los países industrializados no existe un modelo de maquinaria industrial apropiada al procesamiento de las fibras de camélidos sud americanos, han preferido encargar a empresas extranjeras, adaptar las maquinas europeas procesadoras de lana, por lo que son equipos industriales que no llegan al óptimo de su producción, por tanto son de baja productividad. La misma universidad regional tampoco se ha interesado en mejorar, perfeccionar, innovar el procesamiento industrial de la fibra de alpaca. El mayor porcentaje de financiamiento del estado al sector alpaquero se dirige a los micro pequeño y medianos productores de fibra de alpaca, asociación de productores, comunidades campesinas, cooperativas, empresa privada, universidades de la capital (programas como Fincyt II, Fidecom, Fomitec y Fondo mipyme).

La región Arequipa se distingue por un crecimiento sostenido durante diez años con una tasa promedio anual del $6 \%$, la parte débil de este crecimiento es que proviene de los precios altos que alcanzaron materias primas como los minerales, específicamente el cobre que benefició solo algunos sectores de la población. Algunos paradigmas propios de una economía cerrada, como la falta de cooperación, la falta de apoyo para los empresarios que toman riesgo y el centralismo, aún sobreviven, como indicaba Porter, (1995) Arequipa es una región caracterizada por su orgullo y ánimo de independencia del centro, durante el siglo XX tuvo que vivir dentro de una economía cerrada y protegida, con una macroeconomía inestable dirigida por el estado, lo que aumentó la dependencia de los empresarios y arequipeños en general, respecto de las medidas y decisiones del gobierno, fortaleció una cultura de "guardar información", de considerar como "competencia" o "enemigo", a sus proveedores, compradores, al gobierno y a todos en general; se trataba de bajar los costos a expensas de los proveedores o compradores, a pesar de esto, las empresas eran rentables. El nivel de innovación era bajísimo, pero suficiente para una economía cerrada. Con el cambio de modelo de sustitución de importaciones por una economía abierta, los empresarios de Arequipa se vieron obligados a integrarse a la economía y al comercio mundial; a un sistema donde el conocimiento, la tecnología y la forma de hacer empresa evolucionan a gran velocidad. Este es el reto que está enfrentando la economía Arequipeña, que ha obligado a llevar a cabo reestructuraciones empresariales, con el fin de bajar costos y mejorar tecnologías, sin embargo, este proceso que se realiza con lentitud aún no tiene como base una cultura de colaboración, 
desarrollar clúster saludables y eficientes, el de financiar o cofinanciar la innovación de su proceso productivo, en cooperación con otras empresas del sector, con universidades y con distintos niveles de gobierno. Tibiamente algunas empresas (Michell y empresas agroexportadoras), impelidas por la competencia internacional, pues la reproducción de fibra de alpaca se empieza a tener en varios países y la presencia del cashmere (o cachemir) en el mercado mundial, una fibra natural por excelencia, es una de las fibras de cabra más exclusivas y valoradas del mundo; intentan hacer innovaciones unos por cuenta propia y otros en colaboración del estado y de la universidad regional.

Algo semejante sucede con los gobernantes regionales que exponen prioridades muy similares a las del sector privado y también de la academia, lo cual es muy favorable para construir y trabajar en una visión conjunta. La iniciativa de las agendas regionales por parte de la universidad regional, la iniciativa de un parque científico tecnológico por parte de la cámara de comercio y la iniciativa por parte del gobierno regional con el proyecto de innovación "Fortalecimiento y articulación de los actores del ecosistema de innovación y emprendimiento de Arequipa para la competitividad regional", constituyen materiales y fundamentos muy importantes para la colaboración de una triple hélice, como continuación del proyecto de la agenda de innovación inteligente que ha permitido que estén comprometidos las principales organizaciones empresariales académicas y políticas de la región. Es necesario un liderazgo colaborativo, promotor de una visión común entre las empresas, universidades y gobiernos locales, con consenso sobre las metas y prioridades para Arequipa y los roles apropiados para cada componente. En resumen, la falta de cooperación y la discriminación constituyen aún las barreras más importantes para lograr una sostenible triple hélice.

La micro, pequeña, mediana y gran empresa en Arequipa, dedicadas a las actividades de agricultura, minería realizan actividades de innovación al margen de la universidad. En el sector agrícola, dedicada a la exportación de frutales y otros, tradicionalmente son asistidos por la Autoridad Autónoma de Majes (AUTODEMA), en el desarrollo de paquetes tecnológicos adaptados para las zonas productivas, como esfuerzos en innovación. En la actualidad se suman los CITEs y la Universidad, que diseñan innovaciones requeridas por la agroexportación. Las empresas mineras contratan empresas extranjeras que hacen uso de lo último en tecnología, mientras que las empresas locales carecen de normas internacionales técnicas. Más del $90 \%$ de empresarios de la micro y pequeña empresa tienen necesidades de innovación y tecnología, en diseño y capacitación; en investigación y desarrollo, sin embargo, en sus opciones de inversión la relegan al último lugar. Para la mediana y gran empresa sus prioridades se centran en diseño y tecnología no incorporada al capital; el software se considera importante tanto para la producción como para la gestión y afirman que no disponen de presupuesto para capacitar en innovación, reconocen que para ellos es mejor comprar que innovar, por ello no cuentan con personal especializado para la investigación, la innovación y desarrollo de tecnología (Meza, 2017).

En el año 2005, las tres universidades de la región Arequipa firman el documento "Agenda para el desarrollo de Arequipa", como un "compromiso de las universidades por el desarrollo de Arequipa", declarando que "las universidades, como instituciones formativas, les corresponde asumir un rol protagónico en los destinos de los pueblos a través del conocimiento", y "construir una visión compartida de desarrollo" (UCSM, UCSP, UNSA). Durante 15 años, la implementación de la agenda, significó para las tres universidades invertir en capacitar a distintos sectores de la población, sobre: "Internacionalización de la educación superior", "la delincuencia", "debate para el Gobierno Municipal", "inseguridad ciudadana", "talleres de Liderazgo", "foros técnicos", entre otros. Temas indiscutiblemente importantes, pero orientados asumir una responsabilidad enteramente social con la comunidad. Aún falta el compromiso especifico de la universidad de establecer compromisos, convenios y contratos con la empresa regional para hacer realidad su tercera misión. Las tres versiones de la agenda de 2005 al 2018, están alejados aún de la empresa.

La experiencia de la universidad regional con la "Agenda para el desarrollo de Arequipa", la experiencia de "La agenda regional de innovación basada en la especialización inteligente de la región Arequipa 2019-2023", que establece ejes estratégicos de innovación en una economía del conocimiento, articulando a los actores del sistema regional y aprovechando los recursos productivos y principalmente el capital humano avanzado de la región, priorizando la Minería, Agrícola-industrial, Textiles camélidos y Turismo, constituyen fundamentos claves para liderar la triple hélice en la región. Por otra parte, la universidad regional, está avanzando lentamente hacia la formulación de proyectos consensuales en la realización de programas, maestrías y doctorados conjuntos con el más alto nivel académico investigativo. El liderazgo de las unidades de posgrado necesita institucionalizar el emprendedurismo, para dar mayor calidad a la empleabilidad de sus egresados. Las líneas de investigación deben operativizar la visión y metas comunes de la academia, la empresa y el gobierno regional.

La universidad regional, mantiene una visión de una investigación predominantemente académica y disciplinaria: "Aquí tenemos líneas de investigación que va a depender por cada una de las áreas, en lo que es la Maestría en educación superior y gestión de la educación son esas líneas sectoriales; en el lado de historia: historia cultural y política" (Entrev Dir.3, 2018). "las líneas de investigación, se enfocan de acuerdo a 
la maestría o al doctorado que están realizando, entonces podemos decir que las líneas de investigación están en el ámbito Penal, Procesal Penal, Constitucional, pero manteniendo los parámetros de una investigación" (Entrev Dir.4, 2018). "se han establecido líneas de investigación y ellos en lo posible, no siempre, trabajan con esas líneas de investigación que lo han establecido los mismos docentes... es probable que muchas de estas tesis tengan concordancia con la Agenda y otras no" (Entrev Dir.2, 2018)

La necesidad de una cultura de cooperación y de tener y ejercer objetivos comunes se aprecia en los estudiantes de posgrado, quienes van integrándose a las líneas de investigación propuestas por la agenda, solo el $17 \%$ afirma tener información sobre las "Agendas de Desarrollo" a través de los medios de comunicación y redes sociales, un $83 \%$ no tiene conocimiento. A esto se suma que la difusión de las escuelas de posgrado sobre los problemas regionales de Arequipa es mínima; un 22\% de estudiantes han participado en eventos de tal naturaleza y un $78 \%$ no participaron y sostienen que los tres principales problemas en la región son: $34 \%$ transporte, $22 \%$ corrupción y $14 \%$ educación. El $76 \%$ elige como tema de tesis algo relacionado a su experiencia profesional logrado en el lugar donde labora y desde una perspectiva de interés individual, el $24 \%$ lo hace por otras razones, los directivos de las escuelas de posgrado lo confirman: "De lo que yo conozco es que gran parte de los tesistas realizan investigaciones en el ámbito donde ellos operan; si están trabajando en gobiernos locales, sus temas serán de gobiernos locales, si están trabajando en empresa privada también o instituciones públicas como agricultura, ministerio público" (Entre. Dir.1, 2018). "estos documentos (se refiere a las agendas) generalmente no lo conocen los de maestría y doctorado, aunque no se crea, y hacen unos temas que a su mejor parecer dominan, pero en realidad algunos temas que ellos plantean de repente están con estas dimensiones territorial, medio ambiental y todo lo demás, pero no tenemos ese encaje" (Entre. Dir.1, 2018)

Desde el año 2016-2019 la universidad regional produce 3,225 trabajos de investigación, cada institución universitaria tiene políticas fuertemente internas, donde la cooperación interinstitucional e intersectorial es aún débil. Esto se puede apreciar en las siguientes declaraciones de los directivos: "Como estoy poco tiempo en la dirección, todavía he visto más de computación e historia, y que definitivamente está ligada a historia cultural... Tienen ellos (los estudiantes) su propio programa que ellos elaboran y como te digo muchos de ellos van a estar alineado a las líneas matriciales de la universidad como: persona, cultura al eje que la universidad está desarrollando" (Entre. Dir.3, 2018). "hay que ver las líneas de investigación... a nivel de escuela, pero con un alcance más detenido... por ahora no se ha fijado o no se hizo el alcance oficial, significa que está abierta las líneas de investigación, estas se enfocan de acuerdo a la maestría o al doctorado que están realizando" (Entre. Dir.5, 2018).

De esta forma las escuelas de posgrado de la universidad regional aún no están alineada para convertirse en una hélice fundamental de la innovación regional, junto con la empresa y el gobierno. Sin embargo, son los Vicerrectorados de investigación de estas universidades quienes si promueven e impulsan proyectos de investigación en concordancia con las empresas y el gobierno. A nivel interno, las universidades cuentan con estudios generales en todas las especialidades, lo que permite al estudiante tener una formación interdisciplinaria y aprender diversas habilidades sociales, necesarias para relacionarse con la empresa, además se promueve la estrategia de enseñanza basada en problemas y proyectos, lo que está creando condiciones favorables para que la universidad junto con la empresa abran a los estudiantes espacios de prácticas preprofesionales sostenibles, que les permita identificar y resolver los problemas productivos, de mercado, de gestión y las posibilidades de innovación de la empresa y donde intervengan los especialistas de ambas instituciones; esto mismo, contribuirá avanzar en el proceso de cambio de la estructura y organización institucional y del plan curricular que requiere una universidad emprendedora.

La universidad regional cuenta con cuatro escuelas de posgrado con una experiencia de 15 años en promedio, cuenta con investigaciones aportando soluciones a la problemática de la informalidad y de las micro y pequeñas empresas (MYPES) (Rodionov y Velichenkova, 2020), como las siguientes: para las empresas asociadas a la Cámara de Comercio de Industria de Arequipa utiliza de manera indistinta la segmentación demográfica, psicográfica y conductual; otra investigación, indica que el subempleo del sector mype, ha deteriorado el sector manufactura, comercio y sector servicios, recomendando la formalización del sector manufacturero con mayor presencia en el empleo y una tercera investigación refiere promocionar los sectores de comercio y de servicios y los beneficios logrados por las mypes textiles que han sido atendidas por la caja Arequipa que son significativamente mayores, en comparación con las mypes que no han hecho uso de créditos, por lo que recomiendan fortalecer y estimular la micro finanza en las mypes sin distinguir las características que diferencia a las instituciones micro financieras. Finalmente, la región Arequipa produce software para el mercado nacional e internacional, cuenta con grandes recursos de capital social avanzado alrededor de la informática. Según la SUNAT existen 1,490 empresas relacionadas con las TIC que desarrollan Internet de las cosas loT, estas empresas están agremiadas en cinco asociaciones que permite establecer un clúster en este sector (Carvajal et al., 2017). 


\section{DISCUSIÓN}

A diferencia de los países desarrollados, en los países en vías de desarrollo, la participación en proyectos de colaboración entre universidad, empresa y gobierno, enfrenta diversas barreras culturales y brechas organizacionales y normativos que dificultan significativamente la dinámica de una triple hélice. La barrera histórica más inmediata, lo constituía el modelo de sustitución de importación. Con la apertura de la economía se abren mejores posibilidades para la triple hélice, pero este proceso está sujeto a varios factores. En países similares como Chile se tiene muy avanzado la dinámica de la triple hélice y las dificultades se identifican como "errores cometidos en las estrategias", ser "generalistas", "poco selectivas", etc (Planas Serralta \& Fernández de Lucio, 2018). en cambio en Arequipa Perú los problemas son mas profundos como las distancias aún existentes entre la academia y la empresa. Tambien hay algunas coinsidencias, por ejemplo España, donde la dimensión de ser pequeña y mediana empresa se percibe como una gran dificultad para la innovación y el acceso a la innovación (Luengo y Obeso, 2013), en Arequipa-Perú, donde predomina la micro y pequeña empresa, también constituye una dificultad, lo que es agravado por el componente históricocultural, como la discriminación, por lo mismo, es mucho más problemático plantear innovaciones radicales en la región Arequipa; lo que es posible en otros ámbitos donde existen condiciones favorables para la colaboración y el acceso a fuentes de financiación externa. En cambio, las barreras que perciben los directivos para colaborar con investigadores, también están presentes en el caso Arequipa. Igualmente, se puede señalar que el principal elemento que dificulta la cooperación de la Triple Hélice es el presentar visiones y objetivos diferentes entre los distintos componentes de la triple hélice, muy a pesar de la cercanía geográficosocial de los mismos. Los resultados concuerdan con la investigación de Loray (2017), que demuestra que las políticas públicas en el CTI, expresan el perfil del Estado y el desempeño de los actores en función de intereses y visiones predominantes. En el Perú la élite gobernante hasta el 2000 , demostró un perfil y visión de desinterés sobre la CTI y con el retorno de la democracia, fue creciendo su decisión de apoyar la CTI.

La universidad regional tienen muchas condiciones favorables para liderar la dinámica de la triple hélice; el cambio del modelo cerrado de sustitución de importaciones, por un modelo económico abierto ha dado impulso a la investigación, la innovación según la demanda de los productos de exportación tradicional y no tradicional. La universidad para su tercera misión necesita espacios de libre mercado, con una intervención racional del estado, centrado en el financiamiento de las investigaciones, tal como se dan en los países desarrollados; esta situación esta en ciernes, en países como Perú, donde la apertura de mercado se inicia en 1992 y las empresas recién empiezan aprender a competir en el mercado global sobre la base de mejorar la calidad de sus bienes y servicios, haciendo uso de la innovación. La universidad que no tiene una tradición investigativa por tener fundamentos dogmáticos, lejanos a la investigación, lo que explica el bajo prestigio de los investigadores docentes de las universidades.

La apertura del mercado peruano empieza a cambiar ese modelo, que se expresa en la universidad regional, incrementando la cantidad y calidad de investigadores, así como la producción y publicación de paper y la inversión en investigación. La universidad da inicio a la agenda regional y lidera el proyecto de innovación regional; interviene exitosamente en la ejecución de proyectos de investigación e innovación financiados por el estado entre la universidad, empresa, gobiernos locales, permitiendo establecer redes sociales de carácter técnico, entre investigadores de la universidad con especialistas y funcionarios de empresas y con funcionarios públicos y líderes políticos. Promueve la formación de pequeñas empresas de base tecnológica como iniciativa de estudiantes, fortalece los estudios generales que permite dar formación interdisciplinaria en todas las carreras y la estrategia de enseñanza basada en problemas y proyectos, al igual que las escuelas de posgrado y líneas de investigación. La institución más antigua de los empresarios de la región decide la creación del parque tecnológico en alianza con la universidad y el gobierno.

Una de las barreras más significativas que ralentiza la triple hélice regional, es la supervivencia de una cultura de desconfianza, baja cooperación y discriminación, así mismo, para fortalecer los cimientos de un sistema de innovación regional, caso Arequipa, es necesario extender los alcances de impacto de las agendas de desarrollo de una política de responsabilidad social a una política de responsabilidad empresarial, que la universidad involucre a la empresa para que los estudiantes tengan espacios de prácticas sostenibles, que permitan identificar y resolver los problemas productivos de mercado, de gestión y las posibilidades de innovación a ser realizados por especialistas de la empresa y de la universidad. Avanzar a un cambio de la estructura y organización institucional y del plan curricular de la universidad, fortaleciendo su perspectiva emprendedora, lo que permitirá que la universidad lidere la triple hélice, sobre la base de promover una cultura colaborativa regional, comprometiendo a la empresa y a los distintos niveles de gobierno a partir de una visión, objetivos y metas comunes.

\section{CONCLUSIONES}

De acuerdo al trabajo presentado y a los resultados obtenidos, se pueden plantear las siguientes conclusiones principales. El rol de la universidad regional de Arequipa es proactivo en la construcción de la triple hélice, 
abriendo espacios de cooperación con la empresa, principalmente con la pequeña y mediana empresa, realizando eventos, ejecutando proyectos, incrementado el número de patentes, que ayudan en su negociación con las empresas para proyectos de innovación; comprometiendo de diversas formas a los distintos niveles del gobierno regional en la formulación y ejecución de proyectos de investigación, aprovechando el financiamiento del Estado.

Los estudiantes de posgrado a pesar de no conocer ampliamente las propuestas de las agendas realizan investigaciones para sus tesis de grado sobre problemas reales de las empresas preferentemente de las pequeñas y microempresas en relación con las instituciones gubernativas, financieras y empresariales de la región y al mismo tiempo proponiendo alternativas de solución.

El cambio del modelo de sustitución de importaciones por un modelo de economía abierta ha afectado positivamente a la empresa, abriendo nuevas posibilidades de exportación de sus productos no tradicionales y a su vez obligandolas en algunos sectores, especialmente en el sector agro industrial, a realizar investigaciones con la universidad para resolver los problemas que enfrenta con sus exportaciones, teniendo que cumplir algunos estándares de calidad; la universidad por su parte, tiene mejores oportunidades de adquirir equipos y materiales para actualizar y mejorar su infraestructura tecnológica.

La universidad regional ha incrementado significativamente su presupuesto para la investigación, permitiendo el aumento del número de investigadores, de ejecución de proyectos de investigación, aumentar su producción y publicación científica. Así mismo, la publicación de las agendas de desarrollo de Arequipa desde el año 2005, se ha institucionalizado en la región y está permitiendo un acercamiento entre las universidades, los gobiernos y la empresa, contribuyendo de esta forma en la construcción de una visión de desarrollo de la región y contar con líneas de investigación para todas las escuelas profesionales y de posgrado.

Las principales limitaciones que presenta la universidad esta en su lento proceso de cambio de su estructura organizacional y curricular, asi como en la implementación ralentizada de la cultura del aprendizaje en su triple dimensión, haciendo, interactuando y usando (modelo DIU), la falta de una política que establezca centros de práctica permanente en las empresas, que promuevan la innovación para resolver sus problemas productivos, de mercado y de gestión.

De las opiniones que emitieron los directores y estudiantes de las escuelas de posgrado, para realizar sus tesis, resalta que debe darse mas difusión a las propuestas hechas en las agendas regionales y consideran la importancia de la difusión de las agendas, donde los estudiantes puedan tomarlos en cuenta para formular sus investigaciones y contribuir más eficazmente al desarrollo regional.

\section{REFERENCIAS}

Altbach, P. G., Twisted roots: the western impact on asian higher education, https://doi.org/10.1007/BF00138959, Higher Education, 18(1), 9-29 (1989)

Carvajal, R. P., Romero, A. J., y Álvarez, G., Estrategia para contribuir a la implementación de la formación dual de los profesionales de ciencias empresariales en las pequeñas y medianas empresas de la provincia Tungurahua, Ecuador, https://doi.org/10.4067/S0718-50062017000500004, Formacion Universitaria, 10(5), 29-41 (2017)

Consejo Nacional de Ciencia, Tecnología e Innovación Tecnológica-Concytec, I Censo nacional de investigación y desarrollo a centros de invesigación (2016)

Cooke, P., Gomez, M., y Etxebarria, G., Regional innovation systems: Institutional and organisational dimensions, https://doi.org/10.5195/jmla.2019.695, Urban Development and Urban Renewal, 26, 475-491 (1997)

Casas, R. y Mercado, A., Mirada iberoamericana a las políticas de ciencia, tecnología e innovación, 1ํa ed., CLACSO, Buenos Aires (2015)

Del Huerto, M.E., La reforma universitaria de Córdoba: pionera en el legado latinoamericano de universidad popular, Revista Cubana de Educación Superior, 37(1), 37-47 (2018)

Etzkowitz, H., y Leydesdorff, L., The dynamics of innovation: from national systems and "mode 2" to a triple helix of university-industry-government relations, https://doi.org/10.1016/S0048-7333(99)00055-4, Research Policy, 29(2), 109$123(2000)$

Etzkowitz, H., y Leydesdorff, L., The triple helix university-industry-governmet realtions: a laboratory for knowledge based economic development, https://ssrn.com/abstract=2480085, EASST Review, 14(1), 14-19 (1995)

Etzkowitz, H., Mack, A., y otros 3 autores, Innovation by design: SPARK and the overcoming of Stanford University's translational "valley of death" in bio-medicine, https://doi.org/10.1002/mde.2966, Managerial and Decision Economics, 41(6), 1113-1125 (2020)

Frølich, N., Coate, K., Mignot-Gerard, S. y Knill, C., Einheit von forschung und lehre: implications for state funding of universities, https://doi.org/10.1057/hep.2010.2, High Educ Policy, 23, 195-211 (2010) 
Guadián-Fernandez, A., El paradigma cualitativo en la investigación socio-educativa, 1aㅡ ed., Morata, España (2007) Ismodes, A.E., Estudio sobre modelo de oficinas de transferencia tecnológica en el Perú, Concytec, 5, 168 (2015)

Pérez, C.A., Las exportaciones según nivel de contenido tecnológico, Centro de investigación de economía y negocios globales CIEN-ADEX, 1-16 (2019)

Looy, B. V., Debackere, K., y Andries, P., Policies to stimulate regional innovation capabilities via university-industry collaboration: an analysis and an assessment, https://doi.org/10.1111/1467-9310.00293, R and D Management, 33(2), 209-229 (2003)

Loray, R., Políticas públicas en ciencia, tecnología e innovación. Tendencias regionales y espacios de convergencia, https://doi.org/10.7440/res62.2017.07, Revista de Estudios Sociales, (62), 68-80 (2017)

Luengo, M. J., y Obeso, M., El efecto de la triple hélice en los resultados de innovación, https://doi.org/10.1590/S003475902013000400006, Revista de Administração de Empresas, 53(4), 388-399 (2013)

Lundvall, B. A., Higher education, innovation and economic development, Higher education and development, 8, 201-228 (2008)

Lundvall, B. A., National innovation systems - analytical concept and development tool, https://doi.org/10.1080/13662710601130863, Industry and Innovation, 14(1), 95-119 (2007)

Lundvall, B. A., The learning economy and the economics of hope, https://library.oapen.org/bitstream/handle/20.500.12657/31613/626406.pdf?sequence=1, Anthem Press (2016)

Lundvall, B. A., Rasmussen, P., y Lorenz, E., Education in the learning economy: a european perspective, https://doi.org/10.2304/pfie.2008.6.6.681, Policy Futures in Education, 6(6), 681-700 (2008)

Meza, M., Demanda de innovación en la región de Arequipa, Perú. Investigación, Innovación y Desarrollo, 18(1), 11 (2017)

Planas, L.M. y Fernández, I., Primeras estrategias regionales de innovación en Chile, https://dx.doi.org/10.4067/S071827242018000200069, Journal of Technology Management and Innovation, 13(2), 69-81 (2018)

Porter, M., Construyendo las ventajas competitivas del Perú, La región Arequipa, Monitor Company, 24-33 (1995)

Rivera Polo, F., y Rivera Vargas, P., Las universidades regionales ante el desafío de la descentralización. El caso de la Universidad de Aysén en Chile, https://doi.org/10.36857/resu.2018.187.481, Revista de La Educación Superior, 47(187), 49-70 (2018)

Rodionov, D., y Velichenkova, D., Relation between Russian universities and regional innovation development, https://doi.org/10.3390/joitmc6040118, Journal of Open Innovation: Technology, Market, and Complexity, 6(4), 1-26 (2020)

Sagasti, F. R., Superando el síndrome de Sísifo: la experiencia del programa de ciencia y tecnología (FINCyT 1) en el Perú, Nuevas instituciones para la innovación: prácticas y experiencias en América Latina, 193-219 (2014)

Stake, R. E., Investigación con estudio de casos, Ediciones Morata (1998)

Strauss, A., y Corbin, J., Bases de la investigación cualitativa técnicas y procedimientos para desarrollar la teoría fundamentada, Medellín: Universidad de Antioquia, 45 (2003)

Vela Meléndez, L., Acevedo Sánchez, E. R., Yesquen Zapata, P., y Venturra Carrillo, G., Ciencia, tecnología e innovación en el Perú. Necesidad de una política pública descentralista, que institucionaliza las alianzas academiaempresa-estado y sociedad civil, https://doi.org/10.14198/geogra2018.9.106, GeoGraphos, Revista Digital Para Estudiantes de Geografía y Ciencias Sociales, 9, 138-157 (2018)

Yin, R., Investigación sobre estudio de casos, diseño y métodos, 2ª Ed., 5 (2), 1-35, Applied Social Research Methods Series (1994) 